\title{
Domain structures and the influence of current on domains and domain walls in highly spin-polarized $\mathrm{CrO}_{2}$ wire elements
}

\author{
A. Biehler, ${ }^{1}$ M. Kläui,${ }^{1}$ M. Fonin, ${ }^{1}$ C. König, ${ }^{2}$ G. Güntherodt,${ }^{2}$ and U. Rüdiger ${ }^{1}$ \\ ${ }^{1}$ Fachbereich Physik, Universität Konstanz, 78457 Konstanz, Germany \\ ${ }^{2}$ II. Physikalisches Institut, Rheinisch-Westfälische Technische Hochschule Aachen, 52056 Aachen, Germany
}

\begin{abstract}
We present a detailed study of the equilibrium magnetization configurations and their response to injected current pulses in microstructured $\mathrm{CrO}_{2}$ wire elements. Using magnetic force microscopy, we determine that the magnetic domain structure of $\mathrm{CrO}_{2}$ wires strongly depends on the wire geometry, in particular, on the wire width and the wire orientation with respect to the magnetocrystalline anisotropy axes. Depending on the wire geometry and the orientation of the initialization magnetic field used, single domain or closure domain configurations have been observed. The domain widths depend on the wire width and are very well reproduced by analytical calculations, as well as by micromagnetic simulations. Currents of up to $5.1 \times 10^{10} \mathrm{~A} / \mathrm{m}^{2}$ were injected into the $\mathrm{CrO}_{2}$ wires at room temperature and found to alter their magnetic domain configuration. Temperature-dependent resistance measurements during the current pulse injection reveal the importance of Joule heating, which can raise the sample temperature above the Curie temperature of $\mathrm{CrO}_{2}$ for pulses injected at room temperature. Low-temperature magnetoresistance measurements reveal a strong reduction in the domain wall depinning field even for injected current densities as low as $10^{10} \mathrm{~A} / \mathrm{m}^{2}$.
\end{abstract}

PACS number(s): 75.60.Ch, 85.70.Kh, 72.80.Ga

\section{INTRODUCTION}

Highly spin-polarized materials have become the focus of intense research due to their possible applications in spintronic devices ${ }^{1}$ and their exciting magnetotransport properties. $^{2} 100 \%$ spin-polarized materials, where the density of states exhibits a gap in one spin channel, while the other spin channel exhibits metallic behavior, and thus the current is carried exclusively by one spin channel, are commonly called half-metallic ferromagnets.

Spin-dependent transport properties are in general strongly dependent on the spin polarization. Most prominent is the giant magnetoresistance (GMR) effect. ${ }^{3,4}$ Tunneling magnetoresistance (TMR) effects also depend strongly on the polarization of the tunneling electrons, ${ }^{5}$ but here, interface effects play an important role..$^{5}$ Recently, the inverse effect, namely, changes in the magnetization due to the injection of strong currents, has been observed. In multilayer GMR-type pillar structures, the alignment of the two magnetic layers could be switched between parallel and antiparallel due to the spin transfer torque effect when reversing the current direction. ${ }^{6}$ The critical current density was suggested to depend on the spin polarization. ${ }^{7,8}$ In general, interfaces can play a crucial role for magnetoresistance and spin transfer torque effects and often the polarization at interfaces is strongly reduced compared to the bulk value.

In order to make use of the high bulk spin polarization for spin transfer torque effects, single layer elements can be used, where the complications of interfaces are avoided. In such single layer elements, the interplay between currents and domain walls leads to domain wall magnetoresistance, which was predicted to depend on the polarization and the wall spin structure and increases with decreasing wall width. ${ }^{9}$ Strong currents have been predicted to displace and transform domain walls due to the spin transfer torque effect $^{7,10}$ and a number of memory and sensor devices based on this effect have been suggested, a prominent example being the racetrack memory design. ${ }^{11}$

Experimentally current-induced domain wall motion has recently been confirmed. ${ }^{12-19}$ Several important aspects such as domain wall velocities, ${ }^{12,13,18}$ critical current densities, ${ }^{14-16,19}$ thermally assisted motion, ${ }^{17}$ and deformation of the domain wall spin structure due to current ${ }^{13}$ have been addressed. However, the underlying theory of interaction between current and magnetization is still controversial. Two different theoretical approaches have been suggested.

(i) In the ballistic limit, ${ }^{20,21}$ the strength of the spin transfer torque effect, and thus the critical current densities and velocities for wall motion, increases not only with the spin polarization but also with reduced wall width. Thus, in order to increase the domain wall velocities, narrow domain walls which are a consequence of large anisotropies are necessary. ${ }^{9,22}$ Depending on the pinning regime, the critical current densities should also scale with the wall width. ${ }^{23}$

(ii) In the diffusive limit, ${ }^{7,21}$ it is assumed that the spin of the charge carriers follows the local magnetization and this leads to the introduction of an adiabatic torque into the Landau-Lifshitz-Gilbert equation of magnetization dynamics. ${ }^{21,24,25}$ Motivated by large discrepancies between experiment and theory, a nonadiabatic term was also introduced. ${ }^{26,27}$ In the theory of the diffusive regime, both the adiabatic and the nonadiabatic spin torque term depend on the equivalent velocity $\mathbf{u}=\mathbf{J} P g \mu_{B} / 2 e M_{s}$, where $\mathbf{J}$ is the current density, $P$ the spin polarization, and $M_{s}$ the saturation magnetization. The predicted domain wall velocities are directly proportional to $\mathbf{u},{ }^{24,26,27}$ indicating that large velocities can be expected in the materials with high $P$ and small $M_{s}$.

Thus, in order to maximize ballistic and diffusive effects, materials with large anisotropies, as well as with a high spin polarization $P$ and a low saturation magnetization $M_{s}$, should be used. Ferromagnetic $\mathrm{CrO}_{2}$ with a Curie temperature $\left(T_{C}\right)$ of about $393 \mathrm{~K}$ (Ref. 28) is a material that combines large $P$ 
and small $M_{s}$ (resulting in a value $\mathbf{u}$ that is more than four times higher than that of Permalloy) with a large uniaxial anisotropy, making it an interesting candidate for spin transfer torque experiments.

Chromium dioxide $\left(\mathrm{CrO}_{2}\right)$ is a metallic ferromagnetic oxide, which has been widely used as a recording medium in magnetic tapes. $\mathrm{CrO}_{2}$ crystallizes in a tetragonal rutile structure with lattice parameters of $a=b=4.421 \AA$ and $c$ $=2.916 \AA$. The chromium ions are present in a $\mathrm{Cr}^{4+}$ state with a magnetic moment of $2 \mu_{B}$ per ion. Band-structure calculations predict $\mathrm{CrO}_{2}$ to be a half-metallic ferromagnet with $100 \%$ spin polarization of the conduction electrons at $E_{F} \cdot{ }^{29-31}$ This prediction has been validated in several experimental observations, such as point contact Andreev reflection, ${ }^{32-34}$ the Tedrow-Meservey technique, ${ }^{35}$ and spinresolved photoelectron spectroscopy measurements, ${ }^{36,37}$ indicating a very high spin polarization up to $97 \%$ at $1.2 \mathrm{~K},{ }^{35}$ as well as over $90 \%$ at room temperature (RT). ${ }^{37}$

Epitaxial $\mathrm{CrO}_{2}(100)$ thin films grown on $\mathrm{TiO}_{2}(100)$ have an intrinsic magnetocrystalline anisotropy with a pronounced magnetic easy axis parallel to the in-plane [001] direction (c-axis). The experimental observations concerning the magnetic anisotropy of $\mathrm{CrO}_{2}$ (Refs. 28, 38, and 39) have been theoretically reproduced by local spin-density approximation calculations ${ }^{40}$ where the [001] direction of $\mathrm{CrO}_{2}(100)$ films has been identified as the magnetic easy axis and the [010] direction as the magnetic hard axis. The corresponding uniaxial anisotropy constant $K_{1}$ has been determined by several groups to be $19 \mathrm{~kJ} / \mathrm{m}^{3},{ }^{41} 27 \mathrm{~kJ} / \mathrm{m}^{3},{ }^{42}$ or $44 \mathrm{~kJ} / \mathrm{m}^{3}$ (Ref. 28) depending on the structural quality of the films, as well as on their thickness.

In order to observe the influence of current on domain walls in half-metallic ferromagnetic $\mathrm{CrO}_{2}$, we need to be able to reproducibly prepare well separated domain walls. Therefore, the $\mathrm{CrO}_{2}$ films should be microstructured and the resulting magnetic domain configuration of the submicron elements has to be ascertained prior to the experiments with current injection. Only then can the interaction of prepared magnetic structure with injected current pulses be studied in $\mathrm{CrO}_{2}$ elements.

In this work, we investigate the magnetic domain structure and the domain walls in microstructured $\mathrm{CrO}_{2}$ wires with magnetic force microscopy (MFM) at RT. By varying the wire width, the resulting domain configuration is changed. We have determined the combination of wire geometry and initializing magnetic field sequence that yields reproducible and well-defined magnetic domains and domain walls. The domain widths are compared with micromagnetic simulations, as well as analytical theory, and good agreement is found. We have then injected current pulses at RT and observed the effective changes in the magnetic domain configurations and the domain wall positions in the $\mathrm{CrO}_{2}$ wires. The observations are correlated with the induced temperature rise due to the Joule heating during pulse injection. To reduce heating, low-temperature magnetoresistance measurements that reveal the dependence of the depinning field on the injected pulse current density are performed.

\section{EXPERIMENT}

Epitaxial $\mathrm{CrO}_{2}(100)$ films were grown on single-crystal $\mathrm{TiO}_{2}(100)$ substrates by chemical-vapor deposition using

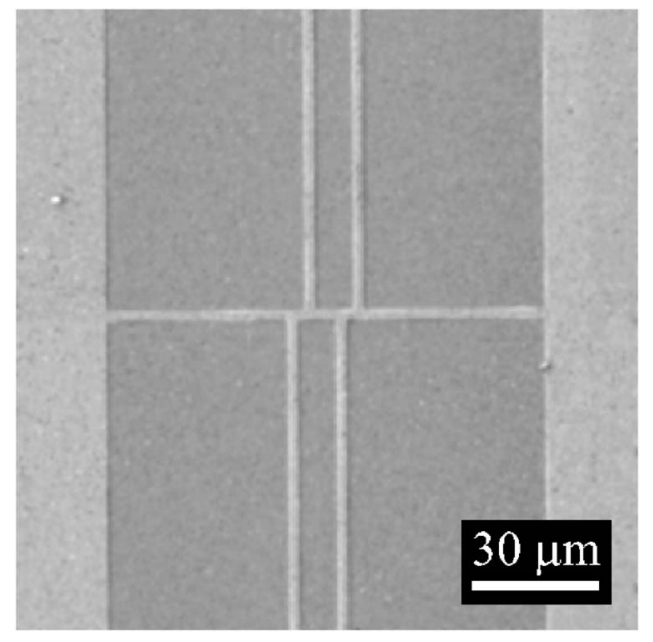

FIG. 1. SEM picture of a $\mathrm{CrO}_{2}$ microstructure: a $2 \mu \mathrm{m}$ wide transport wire (horizontal line) connected to two contact pads (left and right), together with four additional contact leads (vertical lines).

$\mathrm{CrO}_{3}$ as a precursor. The source temperature was kept at $270{ }^{\circ} \mathrm{C}$ within a quartz tube in a one-zone furnace. A heating cartridge implemented in the substrate holder was used to achieve $390-410{ }^{\circ} \mathrm{C}$ at the $\mathrm{TiO}_{2}$ substrate to promote $\mathrm{CrO}_{3}$ decomposition. The sublimed $\mathrm{CrO}_{3}$ precursor is transported by $\mathrm{O}_{2}$ carrier gas flow $\left(1 \mathrm{~atm}, 10 \mathrm{~cm}^{3} / \mathrm{min}\right.$ ) from the source region to the substrate in the reaction zone where it decomposes to form $\mathrm{CrO}_{2}$. Prior to deposition, the $\mathrm{TiO}_{2}$ substrates were cleaned in organic solvents (acetone, isopropanol, and methanol) and subsequently etched in a HF aqueous solution. The typical thickness of the prepared $\mathrm{CrO}_{2}(100)$ films was estimated to be about $60 \mathrm{~nm}$. The described preparation technique was confirmed to yield single-phase epitaxial $\mathrm{CrO}_{2}(100)$ films $\left(T_{C}=385 \mathrm{~K}\right)$ showing a high spin polarization at the Fermi energy at RT. ${ }^{37,43}$ The prepared $\mathrm{CrO}_{2}(100)$ films exhibit an intrinsic uniaxial in-plane anisotropy with a pronounced magnetic easy axis parallel to the [001] direction.

Wires of different widths $(0.5,1,2,3$, and $5 \mu \mathrm{m})$, together with contact leads and pads, were fabricated from epitaxial $\mathrm{CrO}_{2}(100)$ films by conventional e-beam lithography in conjunction with $\mathrm{Ar}^{+}$-ion milling. In some of the structures, notches were introduced, leading to constrictions. Figure 1 shows a scanning electron microscopy (SEM) image of a typical $\mathrm{CrO}_{2}$ microstructure consisting of the main wire with two main contact pads, and four additional contact leads with pads. The main wire presented in Fig. 1 is aligned perpendicular to the [001] direction, i.e., magnetic easy axis, of the $\mathrm{CrO}_{2}(100)$ film. In the following, these structures will be referred to as hard structures whereas the microstructures with the main wires aligned parallel to the [001] direction will be referred to as easy structures.

MFM measurements on the $\mathrm{CrO}_{2}$ wires were performed at RT using a Digital Instruments MULTIMODE ${ }^{\text {TM }}$ scanning probe microscope. Cobalt coated low moment POINTPROBE-PLUS ${ }^{\circledR}$ Silicon-SPM-Sensors from Nanosensors ${ }^{\mathrm{TM}}$ were used as cantilevers in all experiments.

The temperature-dependent measurements were carried out in the range from 4.2 to $300 \mathrm{~K}$ in a liquid- ${ }^{4} \mathrm{He}$ bath 
TABLE I. $\mathrm{CrO}_{2}$ material parameters used in the micromagnetic simulations performed with the OOMMF code.

\begin{tabular}{lcr}
\hline \hline Saturation magnetization $M_{S}$ & $4.75 \times 10^{5} \mathrm{~A} / \mathrm{m}$ & Refs. 45 and 46 \\
Exchange stiffness constant $A$ & $4.6 \times 10^{-12} \mathrm{~J} / \mathrm{m}$ & Ref. 47 \\
Anisotropy constant $K_{1}$ & $2.7 \times 10^{4} \mathrm{~J} / \mathrm{m}^{3}$ & Ref. 42 \\
Damping parameter & 0.0023 & Ref. 48 \\
\hline \hline
\end{tabular}

cryostat magnetotransport setup. The temperature during pulse injection was determined by resistance measurements in a four-point geometry, as detailed in Ref. 19.

Micromagnetic simulations were performed using the object oriented micromagnetic framework (OOMMF) code. ${ }^{44}$ Material constants used for the simulations are presented in Table I. The cell size in the simulation was set to $4 \mathrm{~nm}$, and thus smaller than the Bloch wall width parameter $\sqrt{A / K_{1}}$ $=13.1 \mathrm{~nm}$, with $A$ the exchange stiffness constant and $K_{1}$ the anisotropy constant, and the exchange length $l=\sqrt{A / K_{d}}$ $=5.70 \mathrm{~nm}$ with the stray field energy coefficient $K_{d}$ $=\mu_{0} M_{S}^{2} / 2$.

\section{RESULTS AND DISCUSSION}

We first consider the magnetization configurations in micron-sized $\mathrm{CrO}_{2}$ wires depending on wire orientation with respect to the magnetic easy axis, as well as on the initialization magnetic field sequence used. Figure 2(a) shows an MFM image of a $2 \mu \mathrm{m}$ wide wire fabricated parallel to the magnetic easy axis (easy structure) after applying an initializing magnetic field parallel to the long wire axis. The easy structure is obviously in a single domain remanent state showing MFM contrast only at the artificially introduced constriction where the magnetic stray fields exit and enter the material, which can be detected by the MFM as bright and dark contrasts in close proximity to the constriction. The
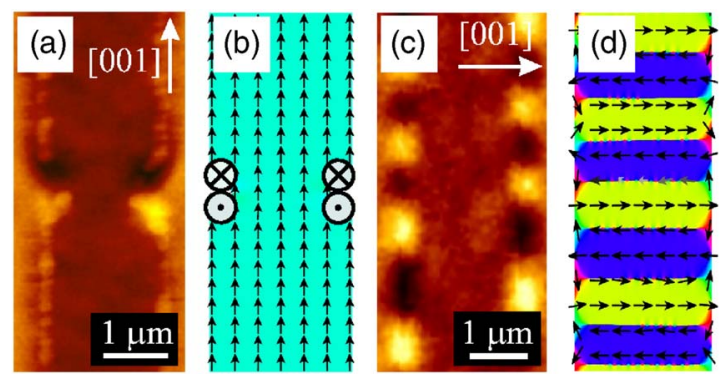

FIG. 2. (Color online) (a) An MFM image of a $2 \mu \mathrm{m}$ wide $\mathrm{CrO}_{2}$ wire (easy structure) with a constriction. Magnetic contrast due to stray fields can only be detected at the constriction. Bright contrast along the edges of the wire is due to topographic contribution from the remaining photoresist. The result of a micromagnetic simulation presented in (b) agrees with the experimental data and furthermore indicates that magnetic flux exits and enters the material only at the constriction. (c) An MFM image showing a demagnetized $2 \mu \mathrm{m}$ wide $\mathrm{CrO}_{2}$ wire (hard structure). The magnetic stray field indicates a closure domain configuration, which is in good agreement with the micromagnetic simulation (d). The local directions of the magnetization in (b) and (d) are marked by arrows.
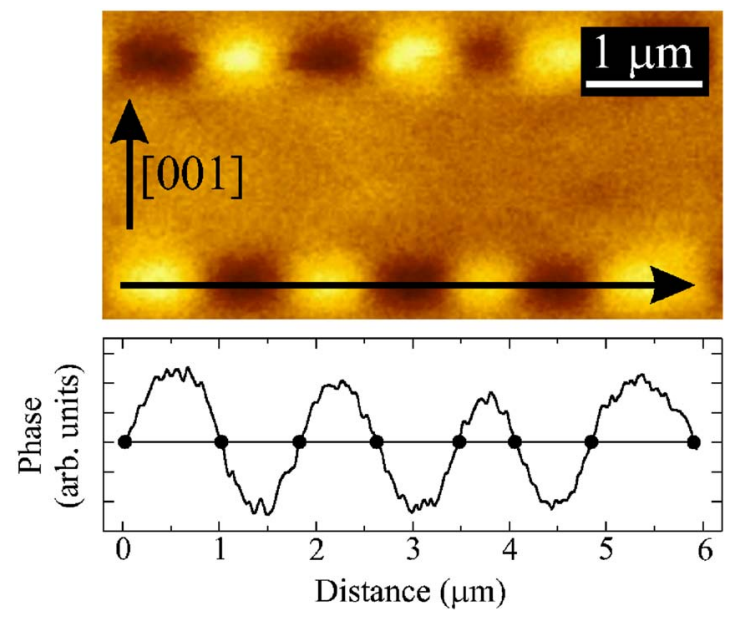

FIG. 3. (Color online) An MFM image of a $2 \mu \mathrm{m}$ wide demagnetized $\mathrm{CrO}_{2}$ wire (hard structure) exhibiting closure domains. Height profile was taken along the horizontal arrow marked in the MFM image. The dots in the height profile mark the borders of the domains and allow an accurate measurement of the domain widths.

bright contrast observed along the edges of the wire is of pure topographic origin due to the remaining photoresist. The resulting single domain remanent state with the magnetization of wires parallel to the magnetic easy axis is observed in the easy structures independent of wire width. In Fig. 2(b), the result of a micromagnetic simulation performed with the OOMMF code is shown, confirming the formation of a single domain state with the magnetization parallel to the [001] direction. The single domain state observed in the easy structures is caused by the dominating magnetocrystalline anisotropy, which aligns the magnetization along the easy axis. Different from the easy structures, the remanent magnetization configuration of the hard structures exhibits a flux closure domain structure with stripes of alternating magnetization parallel to the magnetic easy axis, as presented in Fig. 2(c), which is similar to what has been observed in other materials with a uniaxial anisotropy. ${ }^{49}$ The result of a corresponding micromagnetic simulation performed using the OOMMF code is shown in Fig. 2(d). The simulation was started with a uniform out-of-plane magnetization and then relaxed without an applied magnetic field. The micromagnetic simulation [Fig. 2(d)] shows that the magnetization at the edge of the wire is in fact not completely parallel to the magnetic easy axis but is partially tilted, forming closure domains, in order to reduce the stray field energy, ${ }^{22}$ leading to the magnetic contrast observed in Fig. 2(c). It should be noted that the contact leads adjacent to the wire do not influence the magnetic structure in the wire, which was confirmed by the observation of the same domain structure in the regions of the wire in close proximity to the contact leads and in those that are further away.

Figure 3 shows an MFM image of the $2 \mu \mathrm{m}$ wide $\mathrm{CrO}_{2}$ hard structure, together with an MFM height profile along the horizontal arrow marked in the image. The dots in the height profile mark the positions where the measured phase shift is equal to zero, defining the boundary between two adjacent magnetic domains. The magnetic domain width in the $2 \mu \mathrm{m}$ wide hard structure was determined to be about 

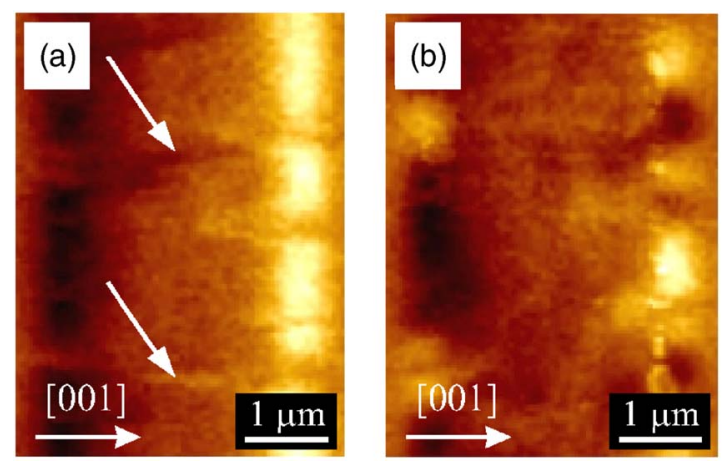

FIG. 4. (Color online) The results of two consecutive MFM measurements on a $3 \mu \mathrm{m}$ wide $\mathrm{CrO}_{2}$ wire (hard structure) after applying an initializing magnetic field parallel to the magnetic easy axis are shown. (a) First, a magnetization parallel to the magnetic easy axis and along the initializing field is detected. The interaction between the magnetic MFM tip and the magnetization in the wire causes the nucleation of closure domains, which manifests itself by horizontal stripes [marked by arrows in (a)]. (b) The next MFM measurement on the same wire region reveals the nucleated closure domains.

$500 \mathrm{~nm}$. However, the widths of the closure domains depend strongly on the wire width, as well as on the orientation of the initialization magnetic field used, varying from several micrometers down to less than $100 \mathrm{~nm}$. After initializing the samples (hard structure) with the magnetic field parallel to the magnetic easy axis, wires with widths larger than $3 \mu \mathrm{m}$ are in a single domain state with the magnetization parallel to the initial magnetic field direction [see Fig. 4(a)]. A closure domain configuration was found for wires with $1 \mu \mathrm{m}$ width or less (hard structures) after application of such an initializing field. For the widths in between, commonly, a combination of both configurations can be found, so that wide domains with the magnetization parallel to the direction of the initializing magnetic field are interrupted by narrow domains with the opposite magnetization. The single domain state in hard structures observed for these wire widths created by reducing the field along the magnetic easy axis can be easily switched to the closure domain configuration by an MFM tip, as shown in Fig. 4. The first MFM measurement after applying the initializing magnetic field reveals a single domain state [Fig. 4(a)]. Horizontal stripes (marked by white arrows) visible in Fig. 4(a) are due to the local switching of magnetization caused by an MFM tip resulting as a consequence in the nucleation of closure domains of opposite magnetization direction. The following MFM measurement on the same wire region [Fig. 4(b)] indicates the formation of a closure domain configuration.

This behavior shows that the state of high remanence (single domain state) in a hard structure is a metastable state, which is separated from the energetically more favorable closure domain configuration by a potential barrier which can already be overcome by the stray field of an MFM tip. This potential barrier is caused by the magnetocrystalline anisotropy energy, which arises when the magnetization rotates from the original easy direction across the perpendicular hard direction to the opposite easy direction. The observed dependence of the magnetization configuration on the wire width
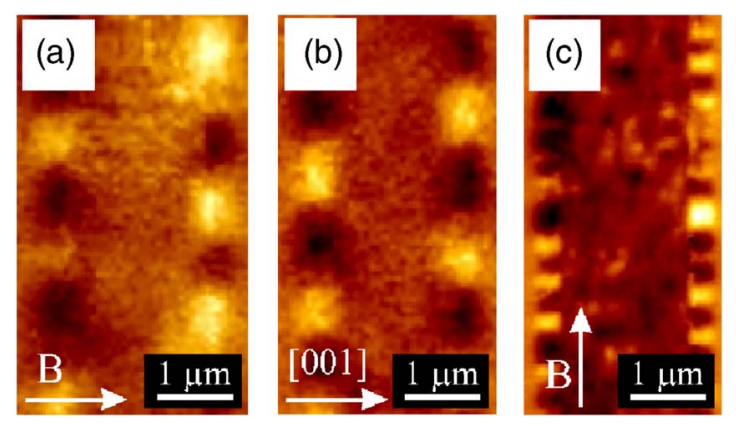

FIG. 5. (Color online) MFM images of a $2 \mu \mathrm{m}$ wide $\mathrm{CrO}_{2}$ wire (hard structure) (a) after applying an initializing magnetic field parallel to the magnetic easy axis, (b) after demagnetization, and (c) after applying an initializing magnetic field perpendicular to the easy magnetic axis are shown.

can be explained by the shape anisotropy contribution which favors the magnetization orientation along the long wire axis, leading to a substantial reduction of the potential barrier height for the narrow wires.

The changes in the magnetic configurations in the hard structures after applying different initializing magnetic fields were studied by MFM and are presented in Fig. 5. After initializing the hard structures with the magnetic field applied parallel to the long wire axis, a less regular pattern of closure domains was observed [Fig. 5(c)]. In this case, closure domains show smaller widths compared with those obtained after applying an initializing magnetic field parallel to the easy axis [Fig. 5(a)] or after demagnetization in an alternating magnetic field with a decreasing amplitude [Fig. 5(b)]. Moreover, some domains do not extend across the whole wire width, leading to the formation of head-to-head or tail-to-tail domain walls inside the wire which can be observed due to the bright or dark MFM contrast within the wire [Fig. 5(c)]. The formation of narrower domains can be explained by different conditions compared with the case of the initializing field applied parallel to the magnetic easy axis. Upon decreasing the initializing magnetic field, the shape anisotropy tries to keep the magnetization in its original direction, while the magnetocrystalline anisotropy tries to rotate the magnetization in the direction of the magnetic easy axis. Since the magnetocrystalline anisotropy dominates in the investigated $\mathrm{CrO}_{2}$ microstructures, the magnetization will align parallel to the magnetic easy axis when the initializing field is reduced.

The energetically favorable magnetization configuration of thin films showing a uniaxial magnetocrystalline anisotropy was studied theoretically by Kittel. ${ }^{50}$ The results of this study apply for the $\mathrm{CrO}_{2}$ wires as well, predicting a regular magnetization pattern of closure domains for the hard structures. Depending on the material constant ${ }^{22} Q=K_{1} / K_{d}$, the width of the closure domains $w$ can be determined as an explicit function of the wire width $D$. For $Q \gg 1$, the open Kittel model ${ }^{22}$ applies. For $Q \ll 1$, the flux closure domains are formed at the edge of the wire (analytical flux closure model). For intermediate values of $Q$, the width of the closure domains has to be determined numerically. Since $Q$ equals 0.19 in the case of $\mathrm{CrO}_{2}$, the flux closure model is expected to apply. This model yields the relation $w$ 


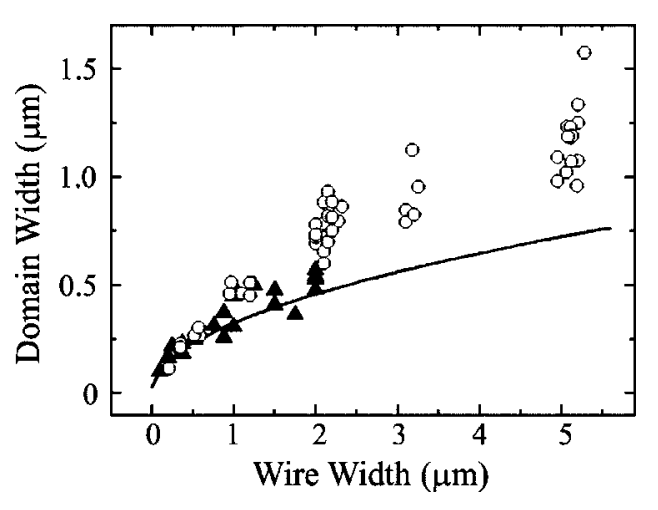

FIG. 6. The measured width of closure domains in a hard structure in the demagnetized state as a function of $\mathrm{CrO}_{2}$ wire width is shown. In addition to the experimental data (open circles), the results of micromagnetic simulations (full up triangles) and the theoretical curve (solid line) according to the flux closure model are presented.

$=2 \sqrt{2 D \sqrt{A / K_{1}}}$ for the energetically most favorable domain width $w .^{22}$ In order to compare experimental results with the theoretical model, the initializing field sequence has to yield the global energy minimum magnetization configuration. For this purpose, the hard structures have been demagnetized by a sinusoidal magnetic field applied parallel to the easy axis with a decreasing amplitude. An MFM image of a demagnetized $2 \mu \mathrm{m}$ wide wire (hard structure) is shown in Fig. 5(b).

Figure 6 shows experimentally measured domain widths as a function of the $\mathrm{CrO}_{2}$ wire width (open circles), together with the theoretical curve obtained on the basis of the flux closure model (solid line). Each experimental data point represents an averaged domain width calculated for one MFM image. Additionally, the results of numerical simulations performed with the OOMMF code for $\mathrm{CrO}_{2}$ wires of different widths are shown (full up triangles in Fig. 6). The parameters of the simulation are listed in Table I. The initial magnetization was chosen to be out of plane and relaxed from this energetically unfavorable state without an applied magnetic field. The micromagnetic simulations were found to be in good agreement with the analytical model, showing only a slight deviation in the region of larger widths of the closure domains. This discrepancy can be attributed to the fact that the analytical model describes a complete flux closure situation $(Q \ll 1)$, while the micromagnetic simulations were performed for the more realistic case of $Q=0.19$. The experimental data shows qualitatively the same behavior compared to both theoretical curves with a quantitative deviation for the larger domain widths, especially at larger wire widths. This deviation can be attributed to the fact that the energetically most favorable magnetization state has not been attained by the chosen initializing field sequence, which means that the total energy could be reduced by the nucleation of further domains. This could be due to defects, such as edge irregularities, which are not taken into account in the simulation and which can pin the magnetization and prevent the nucleation of reverse domains. This is further supported by the large distribution of the measured values at larger wire widths.

In the following, the response of the magnetic structure in the $\mathrm{CrO}_{2}$ wires (hard structures, as well as easy structures) to
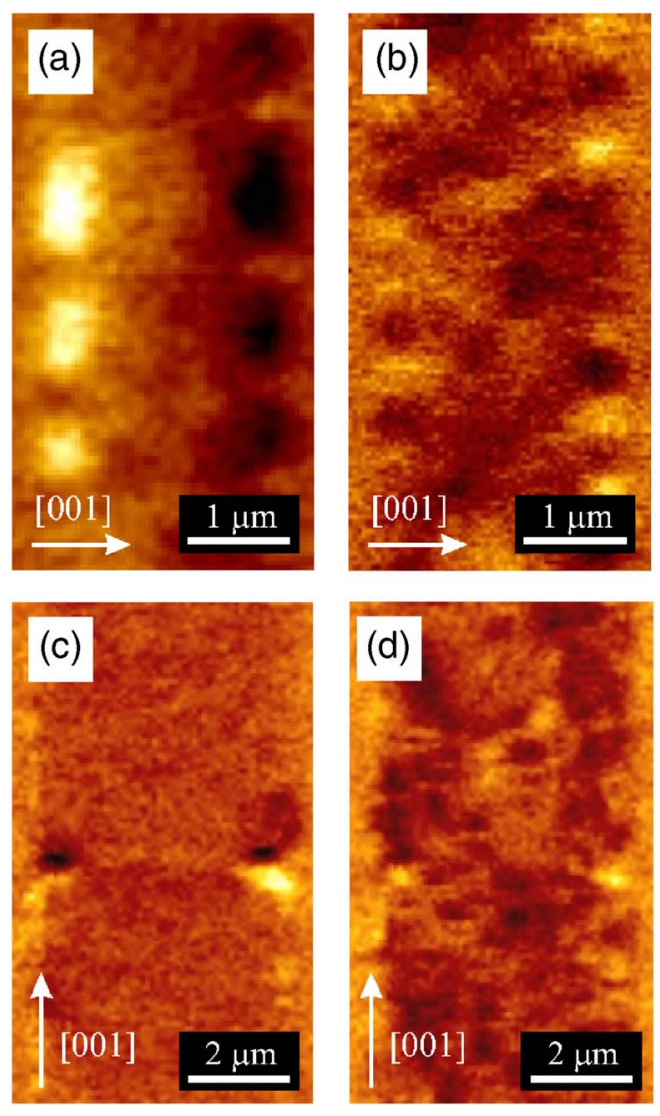

FIG. 7. (Color online) MFM images of a $2 \mu \mathrm{m}$ wide $\mathrm{CrO}_{2}$ wire (hard structure) (a) before and (b) after the injection of a current pulse. The current density was about $5.1 \times 10^{10} \mathrm{~A} / \mathrm{m}^{2}$ and the pulse duration was $10 \mu \mathrm{s}$. In (c) and (d), MFM images of a $5 \mu \mathrm{m}$ wide (easy structure) before and after the injection of a current pulse are shown, respectively. The current density was about 4.7 $\times 10^{10} \mathrm{~A} / \mathrm{m}^{2}$ and the duration of the pulse was $20 \mu \mathrm{s}$.

electrical current was investigated in order to test if spin torque-related effects, i.e., current-induced domain wall motion, can be observed. First, experiments were performed on the hard structures with a well-defined closure domain configuration, which allows an exact observation of a possible domain wall displacement upon current injection. The samples were prepared by choosing an appropriate initializing field and width of the wire. The stability of the magnetization pattern against MFM measurements was confirmed by repeated scanning of the same region of the wire. We observed no change in the magnetic structure of the wires for current densities smaller than $j_{v}=4 \times 10^{10} \mathrm{~A} / \mathrm{m}^{2}$. Around and above this value, the magnetization configuration changed dramatically, as shown for a $2 \mu \mathrm{m}$ wide wire (hard structure) in Figs. 7(a) and 7(b). After a current pulse with a current density of $5.1 \times 10^{10} \mathrm{~A} / \mathrm{m}^{2}$ and a duration of $10 \mu \mathrm{s}$, the MFM measurements still indicate the closure domain configuration, but the domain arrangement does not show any correlation with the domain pattern observed before the pulse injection.

In order to clarify whether a spin transfer torque effect is responsible for the observation, we have also injected current pulses into the easy structures with no domain walls [Figs. 


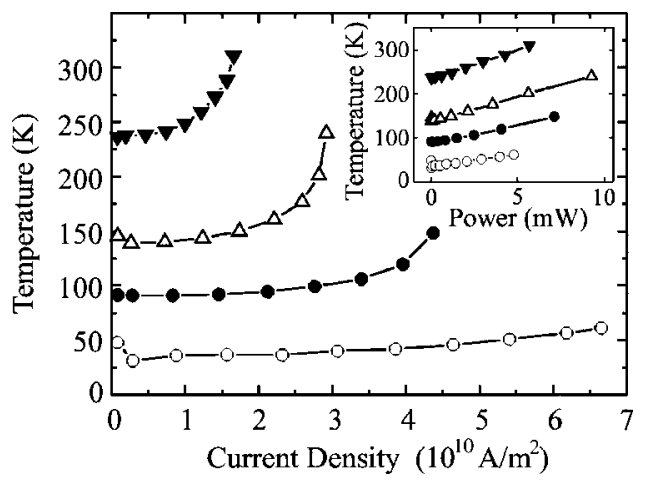

FIG. 8. Temperature of $\mathrm{CrO}_{2}$ wires during the current pulses as a function of current density measured for the initial temperatures of $50 \mathrm{~K}$ (open circles), $100 \mathrm{~K}$ (full circles), $150 \mathrm{~K}$ (open triangles up), and $250 \mathrm{~K}$ (full triangles down). The inset shows the temperature during pulse injection as a function of power at the same initial temperatures.

7(c) and 7(d)]. The magnetization configuration in easy structures is also changed after the injection of current pulses with the density around $j_{v}$, as seen in Fig. 7 for a $5 \mu \mathrm{m}$ wide wire (easy structure). The wire was originally magnetized parallel to the magnetic easy axis and magnetic stray field was only detected at the constriction in the middle. After injection of a pulse with a current density of 4.7 $\times 10^{10} \mathrm{~A} / \mathrm{m}^{2}$ and a duration of $20 \mu \mathrm{s}$, magnetic stray field can be detected above most parts of the wire, indicating the formation of new domains. The change in the nearly uniform magnetization configuration of the easy structure at the same current densities, at which changes in the hard structures occur, rules out pure current-induced domain wall motion. However, together with the domain wall motion, a domain nucleation due to the spin torque effect has also been predicted. ${ }^{51}$ However, the experiments show that no reproducible changes in the magnetization configuration in the hard structures, as well as in the easy structures, upon current injection could be detected.

In order to investigate the reasons for the observed changes further, we measured the temperature rise in a $\mathrm{CrO}_{2}$ wire as a function of the current density at different initial temperatures, as shown in Fig. 8. The temperature of a $\mathrm{CrO}_{2}$ wire increases as a nonlinear function of current density which is due to the higher electrical resistance $R$ of the wire at higher temperatures since the power is given by $P=R I^{2}$ with the current $I$. As expected, the temperature increases nearly linearly as a function of the power, which is shown in the inset in Fig. 8. The heating was found to be quite strong for the relevant current densities and at temperatures above $100 \mathrm{~K}$. At an initial temperature of $250 \mathrm{~K}$, the temperature of the wire increased up to $311 \mathrm{~K}$ during a pulse with current density of $2.1 \times 10^{10} \mathrm{~A} / \mathrm{m}^{2}$. As the temperature rise increases further with increasing initial temperature, we can extrapolate that the wire is heated above $T_{C}$ during pulses with a current density higher than $j_{v}$ if injected at RT. This means that the wire is completely demagnetized after the current pulse and, by cooling down below $T_{C}$, a new magnetization configuration is established. Since the measurements of the resistance of the structures before and after the pulse show
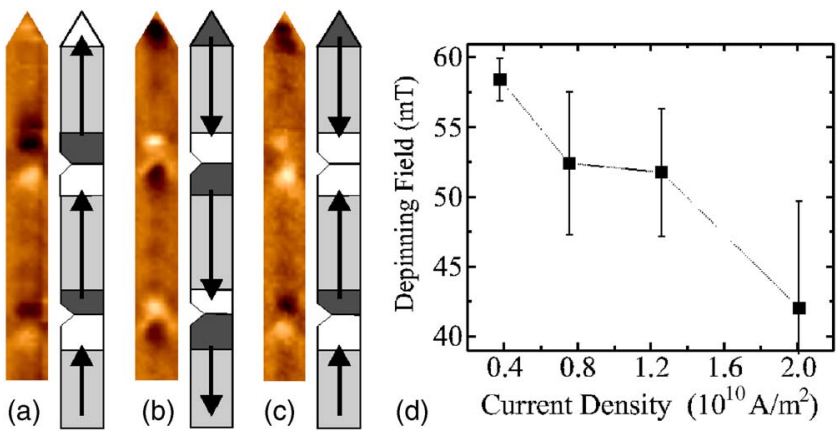

FIG. 9. (Color online) MFM images and corresponding schematic visualization of the remanent magnetization configurations in $1 \mu \mathrm{m}$ wide $\mathrm{CrO}_{2}$ easy structure elements with artificially introduced $500 \mathrm{~nm}$ constrictions. (a) shows the structure after saturation with a $1 \mathrm{~T}$ field upward and (b) shows that after downward saturation $(1 \mathrm{~T})$. (c) After application of $12 \mathrm{mT}$, part of the structure has reversed and a domain wall is pinned at the top constriction. In (d), the domain wall depinning field is presented as a function of injected current density (with electrons flowing in the direction of the wall motion) for an easy structure with a constriction. The field is reduced by a third for a current density of $10^{10} \mathrm{~A} / \mathrm{m}^{2}$.

the same value, we can deduce that the wire is not structurally damaged by the current injection.

To reduce the influence of heating, low-temperature magnetotransport measurements with current injection are necessary. Since in hard structures it is difficult to obtain a single domain wall between two contacts to identify its presence with magnetoresistance (MR), we have used easy structures with artificially introduced constrictions to pin domain walls. To check if a single domain wall can be placed at a constriction, we have first investigated easy structures with constrictions using MFM at RT, as shown in Fig. 9. After saturating the structures with a strong $1 \mathrm{~T}$ field, the elements are all magnetized in the field direction either upward (a) or downward (b). At a field of $12 \mathrm{mT}$, part of the structure reverses and this results in a domain wall that is pinned at a constriction, as seen by the white contrast in Fig. 9(c).

We now use a contacted $2 \mu \mathrm{m}$ wide $\mathrm{CrO}_{2}$ wire with a $1 \mu \mathrm{m}$ constriction and determine the depinning field of the domain wall as a function of the injected current density at $4 \mathrm{~K}$ cryostat temperature ${ }^{19}$ [Fig. 9(d)]. Without a current, the depinning field is around $59 \mathrm{mT}$ and this field starts to decrease if current pulses with a current density of more than $5 \times 10^{9} \mathrm{~A} / \mathrm{m}^{2}$ are injected. For pulse heights of 2 $\times 10^{10} \mathrm{~A} / \mathrm{m}^{2}$, the depinning field is reduced by a third and extrapolation to zero yields a critical current density of 7 $\times 10^{10} \mathrm{~A} / \mathrm{m}^{2}$. The MR signal in these structures is more than 50 times smaller than in Permalloy, which limits the maximum current density that can be injected. ${ }^{52}$ While heating effects are reduced at low temperatures, they are nonetheless present and can also account at least for part of the reduction in the depinning field so that careful further measurements are necessary to completely separate spin torque and heating effects.

\section{CONCLUSION}

We have investigated in detail the remanent magnetization configurations in microstructured $\mathrm{CrO}_{2}$ wires after initializ- 
ing with an external magnetic field and their response to injected current pulses using MFM at RT. The domain structure in $\mathrm{CrO}_{2}(100)$ wires was found to depend strongly on the wire geometry, in particular, the width and the orientation with respect to the magnetocrystalline anisotropy axes. Wires fabricated along the magnetocrystalline easy axis are in a single domain state, while in wires prepared perpendicular to the easy axis, closure domain configurations prevail. Depending on the exact wire geometry, we can stabilize closure domains with different widths by applying initialization magnetic fields along the hard axis and demagnetization fields along the easy axis. Metastable single domain states can be stabilized in the hard structures by applying the initializing field along the easy axis and these states can be switched to closure domains by the interaction with an MFM tip. The domain width of the closure domains depends strongly on the wire width and the measured values are very well reproduced by analytical calculations and micromagnetic simulations. Current pulses of up to $5.1 \times 10^{10} \mathrm{~A} / \mathrm{m}^{2}$ were injected and found to alter the domain configurations for both hard and easy structures. Direct determination of the temperature rise during current pulse injection reveals the importance of the strong temperature dependence of the sample resistance. At elevated temperatures where the sample resistance is highest, Joule heating can increase the sample temperature during pulse injection to above $T_{C}$. This leads to changes in the domain configurations and limits the current densities that can be investigated at RT. Using easy structures with constrictions, we show that domain walls can be pinned at these constrictions. Low-temperature MR measurements reveal that the depinning field is reduced by a third already for current densities as small as $10^{10} \mathrm{~A} / \mathrm{m}^{2}$.

\section{ACKNOWLEDGMENT}

The authors acknowledge support by the Deutsche Forschungsgemeinschaft (DFG, collaborative research center SFB 513).
${ }^{1}$ S. A. Wolf, D. D. Awschalom, R. A. Buhrman, J. M. Daughton, S. Von Molnar, M. L. Roukes, A. Y. Chtchelkanova, and D. M. Treger, Science 294, 1488 (2001).

${ }^{2}$ K. Dörr, J. Phys. D 39, R125 (2006).

${ }^{3}$ M. N. Baibich, J. M. Broto, A. Fert, F. Nguyen Van Dau, F. Petroff, P. Eitenne, G. Creuzet, A. Friederich, and J. Chazelas, Phys. Rev. Lett. 61, 2472 (1988).

${ }^{4}$ G. Binasch, P. Grünberg, F. Saurenbach, and W. Zinn, Phys. Rev. B 39, 4828 (1989).

${ }^{5}$ E. Y. Tsymbal, J. Phys.: Condens. Matter 15, R109 (2003).

${ }^{6}$ J. A. Katine, F. J. Albert, R. A. Buhrman, E. B. Myers, and D. C. Ralph, Phys. Rev. Lett. 84, 3149 (2000).

${ }^{7}$ J. C. Slonczewski, J. Magn. Magn. Mater. 159, L1 (1996).

${ }^{8}$ J. C. Slonczewski, J. Magn. Magn. Mater. 195, L261 (1999).

${ }^{9}$ A. D. Kent, J. Yu, U. Rüdiger, and S. S. P. Parkin, J. Phys.: Condens. Matter 13, R461 (2001).

${ }^{10}$ L. Berger, J. Appl. Phys. 55, 1954 (1984).

${ }^{11}$ S. S. P. Parkin, U.S. Patent No. 6834005 and patent application No. 10/9840552004.

${ }^{12}$ A. Yamaguchi, T. Ono, S. Nasu, K. Miyake, K. Mibu, and T. Shinjo, Phys. Rev. Lett. 92, 077205 (2004).

${ }^{13}$ M. Kläui, P.-O. Jubert, R. Allenspach, A. Bischof, J. A. C. Bland, G. Faini, U. Rüdiger, C. A. F. Vaz, L. Vila, and C. Vouille, Phys. Rev. Lett. 95, 026601 (2005); M. Kläui, M. Laufenberg, L. Heyne, D. Backes, U. Rüdiger, C. A. F. Vaz, J. A. C. Bland, L. J. Heyderman, S. Cherifi, A. Locatelli, T. O. Mentes, and L. Aballe, Appl. Phys. Lett. 88, 232507 (2006).

${ }^{14}$ J. Grollier, P. Boulenc, V. Cros, A. Hamzić, A. Vaurès, A. Fert, and G. Faini, Appl. Phys. Lett. 83, 509 (2003).

${ }^{15}$ N. Vernier, D. A. Allwood, D. Atkinson, M. D. Cooke, and R. P. Cowburn, Europhys. Lett. 65, 526 (2004).

${ }^{16}$ M. Kläui, C. A. F. Vaz, J. A. C. Bland, W. Wernsdorfer, G. Faini, E. Cambril, L. J. Heyderman, F. Nolting, and U. Rüdiger, Phys. Rev. Lett. 94, 106601 (2005).

${ }^{17}$ D. Ravelosona, D. Lacour, J. A. Katine, B. D. Terris, and C. Chappert, Phys. Rev. Lett. 95, 117203 (2005).
${ }^{18}$ M. Yamanouchi, D. Chiba, M. Matsukura, and H. Ohno, Nature (London) 428, 539 (2004).

${ }^{19}$ M. Laufenberg, W. Bührer, D. Bedau, P.-E. Melchy, M. Kläui, L. Vila, G. Faini, C. A. F. Vaz, J. A. C. Bland, and U. Rüdiger, Phys. Rev. Lett. 97, 046602 (2006).

${ }^{20}$ V. A. Gopar, D. Weinmann, R. A. Jalabert, and R. L. Stamps, Phys. Rev. B 69, 014426 (2004).

${ }^{21}$ G. Tatara and H. Kohno, Phys. Rev. Lett. 92, 086601 (2004).

${ }^{22}$ A. Hubert and R. Schäfer, Magnetic Domains (Springer, New York, 1998).

${ }^{23}$ G. Tatara, T. Takayama, H. Kohno, J. Shibata, Y. Nakatani, and H. Fukuyama, J. Phys. Soc. Jpn. 75, 064708 (2006).

${ }^{24}$ Z. Li and S. Zhang, Phys. Rev. Lett. 92, 207203 (2004).

${ }^{25}$ A. Thiaville, Y. Nakatani, J. Miltat, and N. Vernier, J. Appl. Phys. 95, 7049 (2004).

${ }^{26}$ S. Zhang and Z. Li, Phys. Rev. Lett. 93, 127204 (2004).

${ }^{27}$ A. Thiaville, Y. Nakatani, J. Miltat, and Y. Suzuki, Europhys. Lett. 69, 990 (2005).

${ }^{28}$ X. W. Li, A. Gupta, and Gang Xiao, Appl. Phys. Lett. 75, 713 (1999).

${ }^{29}$ K. Schwarz, J. Phys. F: Met. Phys. 16, L211 (1986).

${ }^{30}$ S. P. Lewis, P. B. Allen, and T. Sasaki, Phys. Rev. B 55, 10253 (1997).

${ }^{31}$ M. A. Korotin, V. I. Anisimov, D. I. Khomskii, and G. A. Sawatzky, Phys. Rev. Lett. 80, 4305 (1998).

${ }^{32}$ R. J. Soulen, Jr., J. M. Byers, M. S. Osofsky, B. Nadgorny, T. Ambrose, S. F. Cheng, P. R. Broussard, C. T. Tanaka, J. Nowak, J. S. Moodera, A. Barry, and J. M. D. Coey, Science 282, 85 (1998).

${ }^{33}$ W. J. DeSisto, P. R. Broussard, T. F. Ambrose, B. E. Nadgorny, and M. S. Osofsky, Appl. Phys. Lett. 76, 3789 (2000).

${ }^{34}$ Y. Ji, G. J. Strijkers, F. Y. Yang, C. L. Chien, J. M. Byers, A. Anguelouch, G. Xiao, and A. Gupta, Phys. Rev. Lett. 86, 5585 (2001).

${ }^{35}$ J. S. Parker, S. M. Watts, P. G. Ivanov, and P. Xiong, Phys. Rev. Lett. 88, 196601 (2002). 
${ }^{36}$ K. P. Kämper, W. Schmitt, G. Güntherodt, R. J. Gambino, and R. Ruf, Phys. Rev. Lett. 59, 2788 (1987).

${ }^{37}$ Yu. S. Dedkov, M. Fonin, C. König, U. Rüdiger, G. Güntherodt, S. Senz, and D. Hesse, Appl. Phys. Lett. 80, 4181 (2002).

${ }^{38}$ D. S. Rodbell, J. M. Lommel, and R. C. deVries, J. Phys. Soc. Jpn. 21, 2430 (1966).

${ }^{39}$ A. Gupta, X. W. Li, and G. Xiao, J. Appl. Phys. 87, 6073 (2000).

${ }^{40}$ A. Toropova, G. Kotliar, S. Y. Savrasov, and V. S. Oudovenko, Phys. Rev. B 71, 172403 (2005).

${ }^{41}$ L. Spinu, H. Srikanth, A. Gupta, X. W. Li, and G. Xiao, Phys. Rev. B 62, 8931 (2000).

${ }^{42}$ F. Y. Yang, C. L. Chien, E. F. Ferrari, X. W. Li, G. Xiao, and A. Gupta, Appl. Phys. Lett. 77, 286 (2000).

${ }^{43}$ M. Fonin, Yu. S. Dedkov, C. König, G. Güntherodt, U. Rüdiger, J. Mayer, D. Vyalikh, and S. Molodtsov, Adv. Solid State Phys. 43, 487 (2003).

${ }^{44}$ OOMMF was developed at the Mathematical and Computational Sciences Division at the National Institute of Standards and Technology (NIST) and is freely available at http:// math.nist.gov/oommf/.

${ }^{45}$ H. Brändle, D. Weller, S. S. P. Parkin, J. C. Scott, P. Fumagalli, W. Reim, R. J. Gambino, R. Ruf, and G. Güntherodt, Phys. Rev.
B 46, 13889 (1992).

${ }^{46}$ I. L. Siua, W. F. Egelhoff, D. X. Yang, and H. D. Chopra, J. Appl. Phys. 92, 5409 (2002).

${ }^{47}$ G. Miao, G. Xiao, and A. Gupta, Phys. Rev. B 71, 094418 (2005).

${ }^{48}$ P. Lubitz, M. Rubinstein, M. S. Osofsky, B. E. Nadgorny, R. J. Soulen, K. M. Bussmann, and A. Gupta, J. Appl. Phys. 89, 6695 (2001).

${ }^{49}$ C. L. Dennis, R. P. Borges, L. D. Buda, U. Ebels, J. F. Gregg, M. Hehn, E. Jouguelet, K. Ounadjela, I. Petej, I. L. Prejbeanu, and M. J. Thornton, J. Phys.: Condens. Matter 14, R1175 (2002).

${ }^{50}$ C. Kittel, Phys. Rev. 70, 965 (1946).

${ }^{51}$ J. Shibata, G. Tatara, and H. Kohno, Phys. Rev. Lett. 94, 076601 (2005).

${ }^{52}$ In particular, the MR change due to a domain wall is only a small part of the MR response due to its small volume. A complete magnetoresistive characterization of $\mathrm{CrO}_{2}$ wire structures is beyond the scope of this paper and will be published elsewhere (Ref. 53).

${ }^{53}$ C. König, M. Fonin, M Laufenberg, A. Biehler, W. Bührer, M. Kläui, U. Rüdiger, and G. Güntherodt, Phys. Rev. B 75, 144428 (2007). 